\title{
The Effect of Time-bound Definiteness on Cognitive Learning Performance
}

\author{
Cao Mengyao, Zhang Yufei, Kan Yu, Hu Zeze, Yuan Yuan* \\ School of Sociology and Psychology, Central University of Finance and Economics, Beijing, China
}

Email address:

yuan5701psy@sina.com (Yuan Yuan)

${ }^{*}$ Corresponding author

\section{To cite this article:}

Cao Mengyao, Zhang Yufei, Kan Yu, Hu Zeze, Yuan Yuan. The Effect of Time-bound Definiteness on Cognitive Learning Performance. Psychology and Behavioral Sciences. Vol. 8, No. 4, 2019, pp. 100-105. doi: 10.11648/j.pbs.20190804.13

Received: July 26, 2019; Accepted: September 4, 2019; Published: September 19, 2019

\begin{abstract}
Time is a precious resource that is closely linked to everyone so it is important to play the maximum value of time. Some research show that definite limit of time can affect the attitude to time and then help people to use time efficiently. In order to explore the theoretical significance and practical value of time and time-bound definiteness, this study examined the effect of time-bound definiteness on cognitive learning performance and the mediating effect of time stress. 33 college students were recruited and randomly divided into time-definiteness $(\mathrm{n}=16)$ and time-indefiniteness groups $(\mathrm{n}=17)$, and dependent variable cognitive learning performance was measured by American College Test with controlling attention stability. The results showed that: (1) the time-bound definiteness improved the cognitive learning performance significantly; (2) the mediating effect of time pressure between time-bound definiteness and cognitive learning performance was not significant. The time-bound definiteness could provide a new route to the development of time perception and management, which has academic prospect and deserves to be used in more applied researches. This study provides a remedy for vacancies in the study of time-bound definiteness, and offers valuable suggestions for self-discipline in many areas such as education and economic management.
\end{abstract}

Keywords: Time-bound Definiteness, Time Pressure, Cognitive Learning Performance

\section{Introduction}

Time is a precious resource that people can use, and it is also an important dimension for the body to adapt to the environment. Time gives everyone the same opportunity, but each person's attitude toward time, value of time, processing and planning time are different [1]. The adequacy of time and perception influence the efficiency of individual decision-making [2-3]. How to make full use of time is an important factor affecting learning efficiency [4], and whether there is time-bound definiteness will affect the perception of time [5]. The core of this research is also about the effect of the time-bound definiteness on the individual learning performance.

Time pressure refers to the subjective perception phenomenon that individual has insufficient time or even lack of time. It is not only a cognitive experience in which the individual does not have enough time to do things, but also an emotional experience such as urgency and anxiety [6]. Emotional experience can be long-term or short-term [7]. In short, time pressure is a sense of urgency for individuals to respond to time. For individuals with discrepant differences, time pressure has different positive or negative effect on individual performance. [8] For learning, whether self-regulated learning determines reading performance under time pressure [9-10].

Previous studies have shown that people have personality differences in time perception, and some people will mostly respond in advance at any time interval, while others will respond in error [11], perception and management of time can affect the degree of learning satisfaction [12]. In addition, the stability of attention has also been confirmed to be closely related to the individual's learning performance. Therefore, based on the existing literature, this study focus on controlling the effect of the stability of attention to avoid personality factor errors interfering with experiment results.

There is no research to explore the effect of time-bound definiteness on cognitive learning performance, and the 
experimental results of related study are complex and ambiguous. Existing research has inconsistencies in variables, operational definitions, and experimental operations. It is difficult to compare the results at the same level, which is also the urgent purpose of this study.

Therefore, based on the existing literature, this study focus on whether the individual's time-bound definiteness affects individual learning performance. Murray first proposed the "achievement needs", people with achievement motivations are brave in pursuing high goals in life, not afraid to complete more difficult tasks, and hope that their performance can surpass others. Achievement motivation is the internal driving force of academic success, to some extent, its level of high motivation or low motivation determines the success and failure of students' academic achievement [13]. High achievement motivators have a clear understanding of time, understand the different meanings of time for each person, be good at managing and using time to create more achievements On the contrary, low achievement motivators have chaotic time-management mindset, lack of planning and execution. Frequent failure experiences make them lose control of time [13].

Therefore, based on existing research, this study focuses on the role of achievement motivation in cognitive learning, and whether the differences in achievement motivation will have an effect on cognitive learning performance when time-bound are definite or indefinite.

In theory, the effect of the time-bound definiteness on individual performance has not yet been determined. Cognitive learning is different from perceptual learning, which is a relatively complicated learning process. There are rarely discussion about the performance of individual cognitive learning from the perspective of time-bound definiteness. Individuals can also help their academic performance by controlling whether or not there is a clear time limit. Therefore, this study has important theoretical and practical significance for exploring the time-bound definiteness.

This study hypothesizes that:

Hypothesis 1: The time-bound definiteness can significantly affect cognitive learning performance;

Hypothesis 2: Achievement motivation plays a regulating role in the effect of time-bound definiteness on cognitive learning performance;

Hypothesis 3: Time pressure mediates the path of effect of time-bound definiteness on cognitive learning performance.

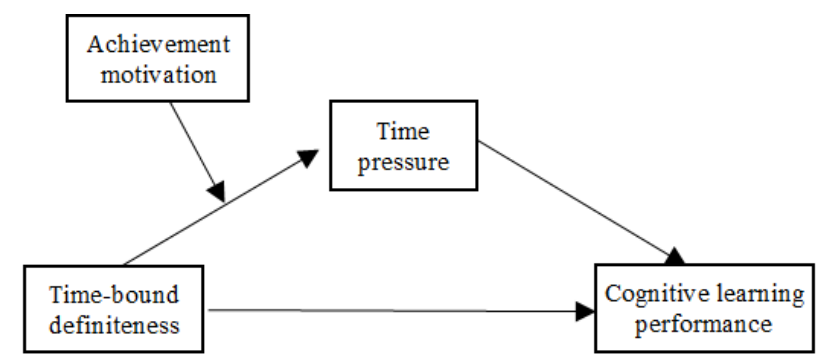

Figure 1. Schematic diagram of the mediating effect model.

\section{Method}

\subsection{Participants}

The subjects were undergraduate students of Central University of Finance and Economics, 33 participants in total (excluding 2 participants), 15 male, 16 female, ages 18-25 years old, normal vision or corrected vision, had not done the same experimental task before. Tthe subjects were Randomly assigned to the experimental group and the control group.

\subsection{Materials}

(1). Reading materials. The reading material in the experiment was adapted from an explanatory text in the ACT test preparation material [14]. The article has a total of 500-600 characters. The article corresponds to 10 multiple-choice questions, and the understanding question and detail question both account for half of the total. Scholars have shown that this experimental material is applicable to Chinese people [15].

(2). Time pressure scale. The time pressure scale was compiled by Svenson (1998) and Wang Dawei (2007) revised time pressure scale. The scale has a total of 8 questions, and 7 questions have been adapted according to the needs of this research, which has high validity.

(3). Achievement motivation scale. The Achievement Motivation Scale (AMS) was compiled by the Norwegian psychologists Gjesme, T. and Nygard, R. in 1970. The Chinese version was translated by Chinese researcher Ye Renmin and Norwegian Hegtvet, K. A. in 1988 based on Chinese traits, and was revised in 1992 in the sample of college students and middle school students. The scale is based on two different tendencies of achievement motivation, with 15 questions for each, and the higher the individual's score in this tendency, the stronger the motivation is [16].

(4). Cancellation experimental materials. Attention stability was measured by Cancellation experiment in the Psykey psychological evaluation system. The cancellation experiment requires the subjects to cross the required numbers of the instructions one by one within the specified time. The experimental material is a randomly distributed digital table with $27 \times 31$ numbers. The participants are required to check and cross out the required numbers from left to right within 3 minutes. The experiment can be used to measure attention stability [17].

\subsection{Design}

Single-factor between-subjects experiment design was used. The independent variable is the time-bound definiteness/indefiniteness, and the experimental group is the time-bound indefiniteness group, the control group is the time-bound definiteness group. The dependent variable is cognitive learning performance, and the observation index is the number of answers and the correct rate of the answer. The control variable has attention Stability, education level, etc. 


\subsection{Procedure}

The experiment was divided into three stages. The first stage and the third stage was completed on written materials, and the second stage was completed on the mobile phone device.

(1). Reading task stage: the experimental group and the control group are required to perform the same task, and there is a stopwatch recording time on the table. Participants in the experimental group were told to perform a reading comprehension task. After the article there were 10 questions, and the questions need to be answered as soon as possible. After "a few" minutes, the experimenter would receive the answer sheet. The control group was told to perform a reading comprehension task. After the article there were 10 questions, and the answer need to be answered as soon as possible. After " 5 " minutes, the experimenter would receive the answer sheet. After the completion of the notification, the experimenter starts to leave the timer, and after 5 minutes, experimenter returns to receive the answer sheet and the timer.

(2). Time pressure measurement stage: The experimental group and the control group were required to use the mobile phone device scan QR code to fill in the time pressure scale.

(3). Achievement Motivation Measurement Stage: The experimental group and the control group were asked to use the mobile phone device scan $\mathrm{QR}$ code to fill in the achievement motivation scale.

(4). Attention stability measurement stage: the experimental group and the control group are required to follow the instructions and perform the Cancellation experiment task. After three minutes, the experimenter returns to receive the answer sheet.

\section{Results}

A total of 33 data were collected for the experiment, of which 31 were valid data. Descriptive statistics of the time pressure, reading completion, and achievement motivation of the subjects and correlation statistics between the variables (Table 1).

Table 1. Descriptive statistics and correlation coefficients $(n=31)$.

\begin{tabular}{lllll}
\hline Variable & $\boldsymbol{M} \pm \boldsymbol{S D}$ & $\mathbf{1}$ & $\mathbf{2}$ & $\mathbf{3}$ \\
\hline Time-bound definiteness & - & - & & \\
Achievement motivation & $2.61 \pm 0.20$ & 0.21 & - & $0.38^{*}$ \\
Time pressure & $2.39 \pm 0.59$ & 0.21 & -0.19 & - \\
The number of completed questions & $6.94 \pm 2.92$ & $0.38^{*}$ & - & $-0.41^{*}$ \\
Accuracy & $0.71 \pm 0.19$ & - & - & - \\
\hline
\end{tabular}

Descriptive statistics for each variable in the experimental and control groups were in Table 2.

Table 2. Descriptive statistics of variables in experimental and control group $(n=31)(M+-S D)$.

\begin{tabular}{|c|c|c|c|c|}
\hline & Time pressure & Mistakes in Cancellation & Completed questions & Accuracy \\
\hline Experimental $(\mathrm{n}=17)$ & $2.28 \pm 0.61$ & $4.12 \pm 5.67$ & $5.94 \pm 3.03$ & $0.69 \pm 0.23$ \\
\hline Control $(n=16)$ & $2.53 \pm 0.57$ & $3.21 \pm 4.48$ & $8.14 \pm 2.35$ & $0.73 \pm 0.13$ \\
\hline
\end{tabular}

The t-test was performed on the number of cancellation errors in the experimental group and the control group. The results showed that the difference in attention stability between the experimental group and the control group was not significant $(\mathrm{t}(29)=0.48, \mathrm{p}=0.632,95 \% \mathrm{CI}[-2.91,4.72]].) . \mathrm{t}$ test was performed on the correct reading rate of the experimental group and the control group. The results showed that there was no significant difference in the correct reading rate between the experimental group and the control group $(\mathrm{t}$ $(29)=0.49, \mathrm{p}=0.628,95 \%$ CI $[-0.18,0.11])$.

The effect of time-bound definiteness on the cognitive learning performance: the t-test on the number of readings in the experimental group and the control group showed that the number of readings in the experimental group was significantly less than that in the control group ( $\mathrm{t}(29)=-2.22$, $\mathrm{p}<0.05,95 \%$ CI $[-4.23,-0.17]$ ) (Figure 2). Since there is no significant difference in the reading accuracy between the experimental group and the control group, the learning performance of the control group is significantly better than that of the experimental group, and hypothesis 1 is supported.

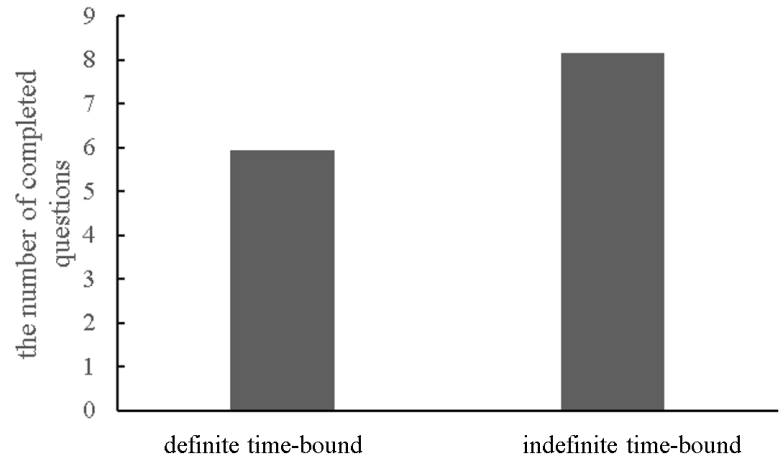

Figure 2. Statistical analysis of cognitive learning performance of the experimental group and the control group.

The mediating role of the time pressure and the regulating role of achievement motivation: First, set the time-definiteness group to the dummy variable, then center the group and the achievement motivation, after that multiply the two to form the product term. According to the process proposed by Wen Zhonglin, Liu Hongyun and Hou Jietai [18], there is a mediating effect of regulation. The results are shown in Table 3 
Table 3. Relationship between time-bound definiteness and learning performance: a mediating effect of regulation.

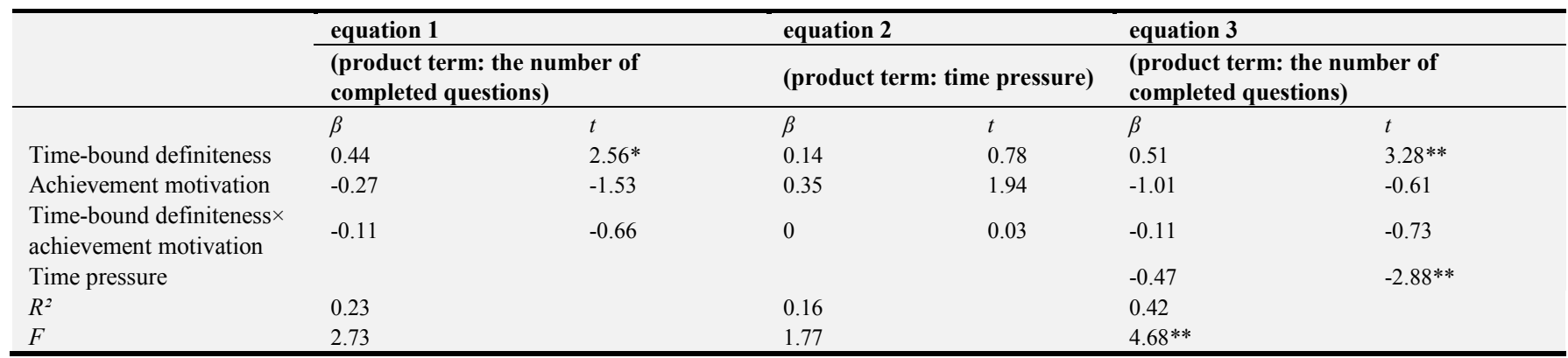

Note: $* * p<0.01, * p<0.05$.

The regression coefficient of the in Equation 1 is significant $(\beta=0.44, t=2.56, p<0.05)$, indicating when the time-bound is definite, the participant answers more reading comprehension questions, and the product term coefficient of achievement motivation and time-bound definiteness is not significant $(\beta=$ $-0.27, \mathrm{t}=-1.53, \mathrm{p}=0.52$ ), indicating there is no interaction. The regression coefficient of product term and time-bound definiteness in Equation 2 is not significant, indicating that time pressure is not significant as a mediator. In summary, the mediation model with regulation is not significant and does not support Hypothesis 2 and Hypothesis 3.

\section{Discussion}

\subsection{About the Effect of Time-bound Definiteness on Learning Performance}

This research starts from the difference between the time-bound definiteness and indefiniteness, revealing that the definiteness of the time limit will significantly affect the performance of cognitive learning. Specifically, after controlling the attention stability and education level of the subjects, the subjects who received the fuzzy time limit of a few minutes did less reading comprehension than the subjects who received the clear time limit of 5 minutes $(\mathrm{t}(29)=-2.22$, $\mathrm{p}<0.05,95 \%$ CI $[-4.23,-0.17])$, but the correct rate was not significant $(\mathrm{t}(29)=0.49, \mathrm{p}=0.628,95 \% \mathrm{CI}[-0.18,0.11])$. This is consistent with the hypothesis of this research. Previous literature did not study the relationship between time-bound definiteness and individual performance, but Ren Jie pointed out in his article that individuals will show more rational behavior under conditions of sufficient time or clear information [19]. The participant with clear time limit may plan the allocation of time in a limited time, and make the choice of profit maximization before the deadline, which is consistent with the research results of this paper. For most people in the real world, it is also a very good choice to improve the enthusiasm and learning effect of individuals or groups by adding a definite time limit, which can improve and optimize the learning system in a clear situation.

In this research, the achievement motivation is used as the regulating variable. The achievement motivation and the number of reading questions and their centralized product terms are tested in the experimental group and the control group. The coefficient of the product term is not significant ( $\beta$ $=-0.11, \mathrm{t}=-0.66) ., \mathrm{p}=0.52)$. The possible reason is that the participants have higher similarity and the difference in achievement motivation is much little $(\mathrm{M}=2.61, \mathrm{SD}=0.20)$. However, the study found that regression analysis of achievement motivation and time pressure showed significant results $(\beta=0.38, t=2.22, p<0.05)$. A possible explanation for this result is that people with high motivations have higher expectations when they complete reading tasks, which leads to a more acute perception of time. The 5-minute time limit makes them feel that time is not enough to complete their expectations. Further research found that under the conditions with clear time limits, participants with high achievement motivation would complete fewer reading questions $(\beta=-0.52$, $\mathrm{t}=-2.09, \mathrm{p}=0.06$ ). The possible reason is that the participants with high motivation are not willing to give up the current answering questions at the end of the time. They want to ensure the accuracy of their own questions, and they will not choose to quickly answer unfinished questions. At the end of the time, the participants with low achievement motivation will be more inclined to quickly answer the unfinished questions to ensure that all the questions have been completed at the end of the time, regardless of the correct rate.

In this research, time pressure was used as a mediator variable, and the time pressure of the experimental group and the control group was tested by t-test. The results showed that the time pressure of the experimental group and the control group was not significant $(\mathrm{t}(29)=-1.18, \mathrm{p}=$ $0.247,95 \%$ CI $[-0.68,0.18])$. According to the existing literature research, with the increase of time pressure, the cognitive load of decision makers under time pressure is higher, and the individual's cognitive processing ability is limited, resulting in a decrease in the amount of information processing [3]. The possible reason for the insignificant time pressure is that the enabling of time pressure on the participants is not obvious enough. The experimenter should remind the participants to pay attention to the clock on the table at the moment to start enabling time pressure. At the same time, the effective data of the experiment was 31 , and the sample size was not large. The above two explanations are the possible reasons why time pressure is not significant as a mediator. 


\subsection{Research Innovation and Limitations}

The innovations of this research mainly include the following aspects:

(1). This experiment focuses on hotspots, conducts time pressure as a mediator, closely follows the upsurge of psychological research, and analyzes the effect of time pressure on learning efficiency objectively and systematically;

(2). This study carefully considers the probabilities of the results from the beginning of the experimental design. The independent variables are selected to be the close to real life, the learning efficiency of the dependent variables, the observation indicators have better measurement standards, and the results obtained are more likely to apply in real work and study experience;

(3). This study combines experimental results with actual conditions, and has practical reference value for practical teaching, learning, and work, and plays a part in the future literature research.

However, there are still some shortcomings in this study. The main limitation is that the enabling of time pressure is not obvious. When the participants complete the learning task, the participants may not care about the clock placed on the table. The solution that came up was to emphasize in the instruction that the participants should pay attention to the clock next to it. Future research will be more refined on the time-bound definiteness and time pressure, and more participants will be recruited to reduce errors due to less statistics.

\subsection{Research Outlook}

Time-bound definiteness is a common independent variable in life, and there is still a lot of research space. In this experiment, although time pressure as a mediator is not significant, it should still attract people's attention. Appropriate achievement motivations help people learn, while excessive achievement motivations have a negative effect. In the field of economic management, managers want to understand the mechanism of time pressure, seek more efficient incentives for employees, and care about changes in psychology and behavior under time pressure. In the field of pedagogy and psychology, we can also use the description and discussion of time-bound definiteness and time pressure to find ways to achieve higher learning efficiency in the teaching situation, form a moderate achievement motivation, and make full use of time-bound definiteness and time pressure to conduct an assistant teaching.

\section{Conclusion}

(1) The time-bound definiteness improved the cognitive learning performance significantly;

(2) The mediating effect of time pressure between time-bound definiteness and cognitive learning performance was not significant.

\section{References}

[1] Huang Xiting, Zhang Zhijie, Feng Sihai, Guo Xiuyan, Lu Houchao, Chen Ying. (2005). A new exploration of time psychology. Psychological Science, 28 (6), 1284-1287.

[2] Chen Jun. (2009). The influence of attribution style and time pressure on decision information processing. Psychological Science (6), 1445-1447.

[3] Yan Xinjing, \& You Xuqun. (2017). The impact of working memory load on the risk decision framework effect. (Doctoral dissertation).

[4] Rubinstein, A.. (2013). Response time and decision making: an experimental study. Judgment and Decision Making, 8 (5), 12.

[5] Tonietto, G., Malkoc, S. A., \& Nowlis, S.. (2018). When an hour feels shorter: future boundary tasks alter consumption by contracting time. Social Science Electronic Publishing.

[6] Devoe, S. E., \& Pfeffer, J.. (2011). Time is tight: how higher economic value of time increases feelings of time pressure. Journal of Applied Psychology, 96 (4), 665-676.

[7] Szalma, J. A., Hancock, P. A., \& Quinn, S. (2008). A meta-analysis of the effect of time pressure on human performance. Human Factors \& Ergonomics Society Annual Meeting Proceedings, 52 (19), 1513-1516.

[8] Keren, G., \& Wu, G.. (2015). 18. Time-pressure Perception and Decision Making. The Wiley Blackwell Handbook of Judgment and Decision Making. John Wiley \& Sons, Ltd.

[9] Liu Rude, Chen Qi. (2000). The mediating influence of on-the-spot self-regulated learning activities on learning outcomes in different situations. Acta Psychologica Sinica, 32 (2), 197-202.

[10] Cone, J., \& Rand, D. G. (2014). Time pressure increases cooperation in competitively framed social dilemmas. PLoS ONE, 9 (12), e115756.

[11] Huang Xiting. (2004). On Time Insight. Psychological Science, 27 (1).

[12] Zhang Zhijie. (2005). Time Management Tendency and Self-esteem, Self-Efficacy, Learning Satisfaction: Analysis of Mediating Roles. Psychological Science, 28 (3).

[13] Zhong Hui. (2003). Research on the relationship between time management tendency and achievement motivation of college students. Psychological Science, 26 (4), 747-747.

[14] Rawson, K. A., Dunlosky, J., Thiede, K. W.. (2000). The rereading effect: metacomprehension accuracy improves across reading trials. Memory \& Cognition, 28 (6), 1004-10.

[15] Zeng Xuli. (2009). The impact of abstract and self-question on the accuracy of meta-understanding. Zhejiang Normal University.

[16] Sun Liyang. (2013). A Study of College Students' Subjective Well-being and Achievement Motivation_-Taking Nanyang Normal University as an Example. Theory of Learning (14), 269-270.

[17] Zhou Huafa. (2010). Research on the relationship between college students' attention stability and self-control and emotional stability. Zhangzhou Teachers College. 
[18] Wen Zhonglin, Liu Hongyun, Hou Jietai. (2012). Analysis of regulation effects and mediating effects. Beijing: Education Science Press.
[19] Ren Jie, Jin Zhicheng, Du Jiahui. (2009). The role of information ambiguity and time adequacy in social dilemma decision-making. Psychological Research, 02 (6). 